\title{
Which sign is more visible? Measuring the visibility of traffic signs through the conspicuity index method
}

\author{
Thomas Porathe $\cdot$ Lennart Strand
}

Received: 8 July 2010 / Accepted: 4 April 2011 / Published online: 7 May 2011

(C) The Author(s) 2011. This article is published with open access at SpringerLink.com

\begin{abstract}
Purpose A new method of measuring visibility taking context into account introduced by Alexander Wertheim has been validated in this study. The purpose was to see if a simple and practical technique could render robust and useful quantitatively measurable results.

Methods An ordinary traffic sign, placed against different backgrounds, was used as a target. Subjects were told to fixate the sign and then slowly deviate with their gaze until they could no longer see the sign in the corner of their eye. This angle was then used as an index for conspicuity. A simple paper and pen method was tested as well as a computer based method.

Results Results for the paper and pen method showed low variance among subjects and a clear correlation between the conspicuity index and intuitive judgments of the visibility of the signs against different backgrounds. For the computer based method the variability was higher but the results still significant between the two groups of dynamic versus static signs.

Conclusions The conclusion is that the conspicuity index method is a simple and useful method that renders quantitative measurements of the visibility of targets taken context into account.
\end{abstract}

Keywords Conspicuity · Visibility · Information design · Traffic signs

\section{T. Porathe $\cdot$ L. Strand $(\bowtie)$}

School of Innovation, Design and Engineering,

Mälardalen University,

Box 325, SE-60531 Eskilstuna, Sweden

e-mail: lennart.strand@mdh.se

T. Porathe

e-mail: thomas.porathe@mdh.se

\section{Introduction}

Traffic signs need to be visible, in strong sunshine, in heavy snowfall, on dark rainy nights etc. And they need to be visible on open country roads and in heavy city traffic. This we know. But how do we make sure, as much as this is possible, that a traffic sign is seen (Fig. 1)?

Asking a number of people is one, subjective, way. A possibly more objective way is the use of a method suggested by the Dutch psychologist Alexander Wertheim. In 1989 he introduced the term conspicuity to denote visibility of an object taking context into account. He defined conspicuity as the extent to which the object, when viewed peripherally, is visually masked by its embedding surroundings. He also suggested a method of measuring conspicuity by determining the angle between the gaze point and the target object and using the angle where the object no longer can be detected as an index for conspicuity [1].

This paper presents some simple studies that were conducted to validate the method and finally also use it on a project designing visible school bus signs.

Thinking about the problem makes us realize certain things. First that visibility is often thought of as attributes to the sign itself, such as color and contrast. Secondly that the context of the sign might be just as important, that the traffic sign might appear in a context which helps to make the sign more prominent but which might also disguise the sign. In Fig. 2 the different signs need to compete with each other in the traffic environment. This complicates the situation.

Look at the images in Fig. 3. In one drawing we see a traffic sign against a green forrest. We might agree that the sign is very conspicus. In the other image we see exactly the same sign in a different context, against a wall in the 

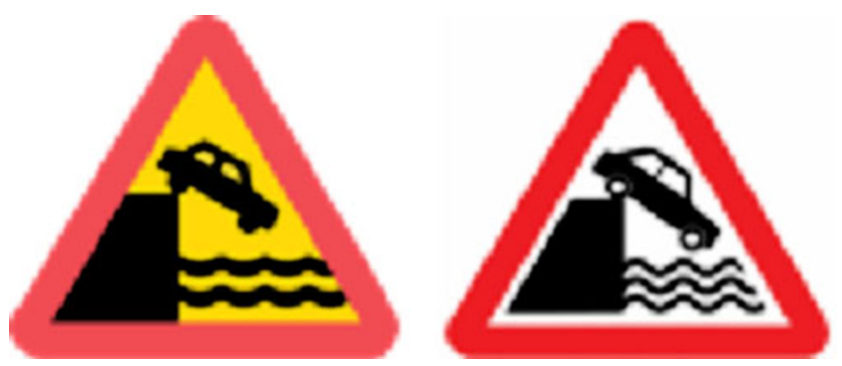

Fig. 1 Here are two European traffic sign warning for quayside or river bank ahead. Which one is more visible? Can we measure a difference? This article presents a new method of measuring visibility

same colors and cluttered with distracting posters and similarlooking signs.

\section{Theoretical background}

The term visibility is often tied to properties of the object itself such as size, color, contrast and brightness. A search on "high visibility clothing" results in numerous hits on bright yellow-and-orange jackets. However, they are only highly visible if the surrounding context is not colored the same way.

The Dutch psychologist Alexander Wertheim suggested in 1989 the term conspicuity to denote visibility of an object taking context into account. He defined conspicuity as the extent to which the object, when viewed peripherally, is visually masked by its embedding surroundings. He also suggested a method of measuring conspicuity by determining the angle between the gaze point and the target object and using the angle where the object no longer can be detected as an index for conspicuity [1].

Receptors in the human eye detect incoming rays of light, which carry information of the world outside. This information is processed by different pathways in the brain. We call this bottom-up processing. Simultaneously, a topdown process governed by experience, expectations, and knowledge helps interpret the sensory information. This is what we call perception.

Our intake of information through the eyes is limited to a number of fixations per second. Between the fixations the gaze moves rapidly over the scene in saccades during which information intake is suppressed. In each fixation we only see sharply in a narrow cone of about $1-2^{\circ}$. The extent of the human peripheral sight amounts to more than $180^{\circ}$ but the quality is rapidly decreasing with increasing angle from the line of sight, both due to decreasing density of photo receptors in the retina and because the receptors are increasingly covered by retinal ganglion cells that scatters the light that fall on them. In just $6^{\circ}$ of eccentricity, the visual acuity is reduced by $75 \%$ [2]. Still the amount of visual information gathered is estimated to be in the range of $10^{8}$ bits per second [3]. Of the massive amount of information that each second hits our eyes only small parts travel up the optic nerve to the visual cortex and only fractions reach our conscious level. We sometimes believe that we see everything around us but phenomena like change blindness [e.g. 4] dramatically reminds us that we are wrong. So how does perception pick out what objects to attend to and process up to our conscious level? How come our traffic sign is so much easier to see against a green forest than against the cluttered wall?

The prevailing Feature Integration Theory by Anne Treisman [5] states that perception picks up objects in a two-step process where a first, fast, unattended, automatic, and parallel process codes a number of separable dimensions of the visual scene such as color, orientation, spatial frequency, brightness and direction of movement. These features are then processed in different areas of the brain and then in a second stage "glued" together to coherent objects by the focal attention in a slow serial process. If in a visual search process we want to detect a target based on just one of these separable features, such as looking for a red line among black lines (in Fig. 4), this could be automatically conducted by the first parallel process and the number of distracters will have little influence on the search time. The target immediately "pops-out."

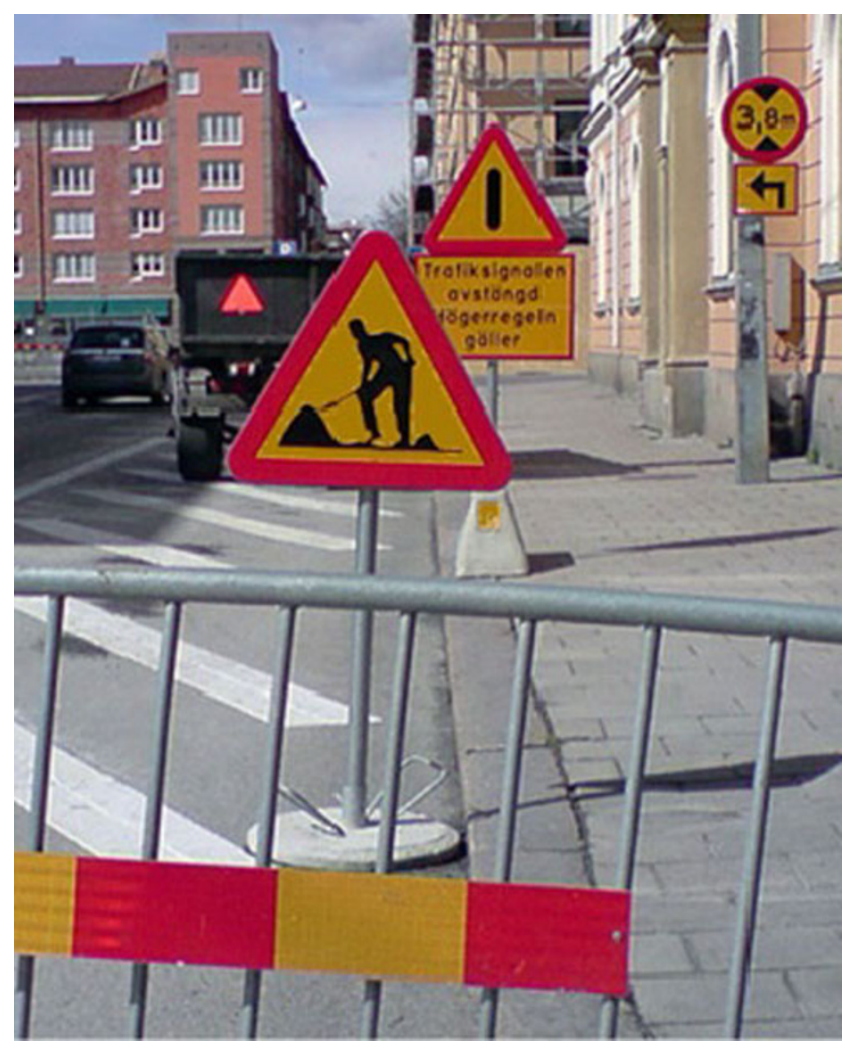

Fig. 2 Each traffic sign is in itself highly visible, but in this competitive environment the conspicuity of each sign is diminished by the other signs 
Fig. 3 The same traffic sign against a background of a green forest or a cluttered wall containing the same colors as the traffic sign itself. Intuitively we can easily say that the sign against the green background is more conspicuous than the other one. But is the difference measurable with the suggested method?
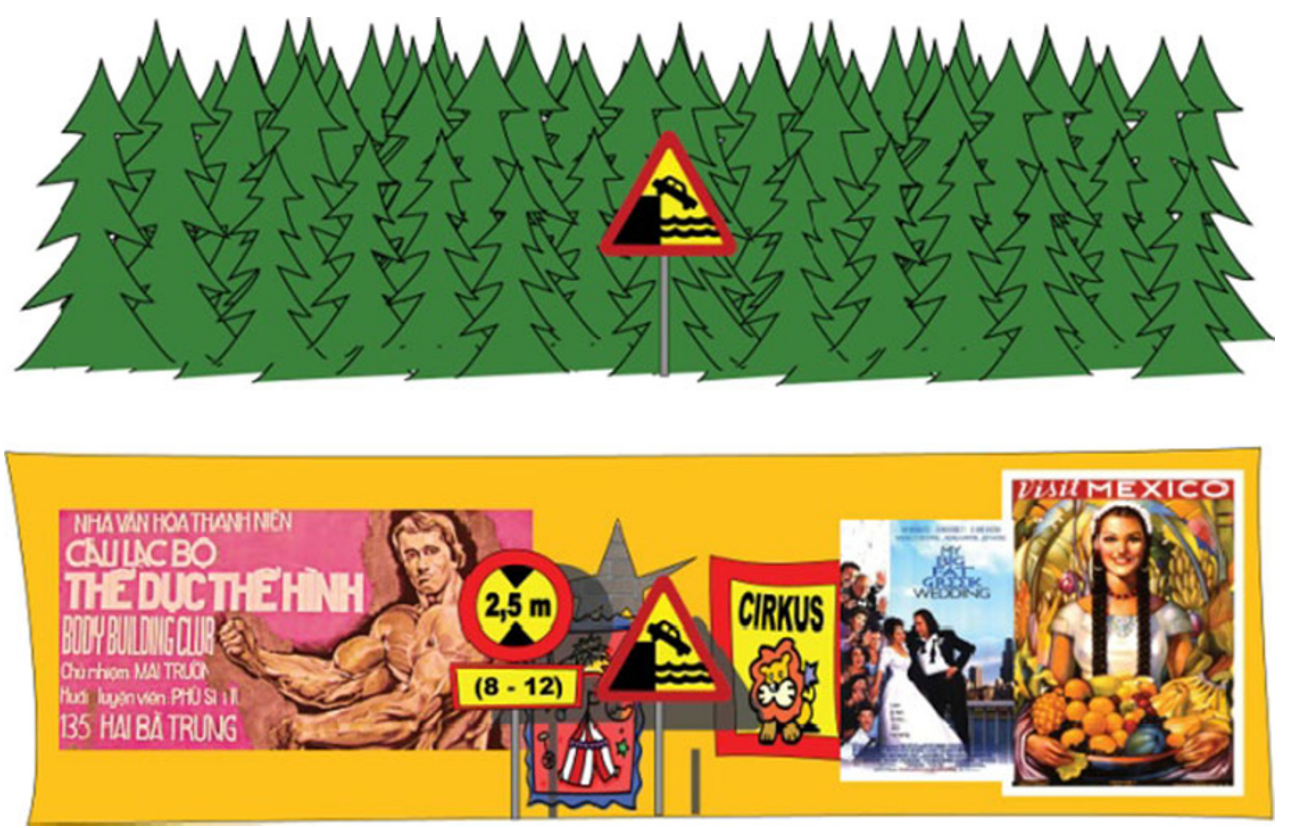

If, on the other hand, a search process involves integrating separable features, the second focused attention mechanism needs to be called in, which involves slow serial search. This can be intuitively demonstrated by searching for the red horizontal line among the red vertical and black horizontal and vertical distracters in the panel of Fig. 5.

In the discussion of what accomplishes the early preattentive selection, Koch and Ullman [6] introduced the idea of saliency maps as a concept of mental twodimensional diagrams that encode the saliency of the visual environment based on the aforementioned features. The most salient feature would get the attention first and so on. It would then be the saliency of the red horizontal line in Fig. 4 that makes it pop-out in the fast pre-attentative stage. Predictions based on automatic saliency maps made by computers would then be possible to make.

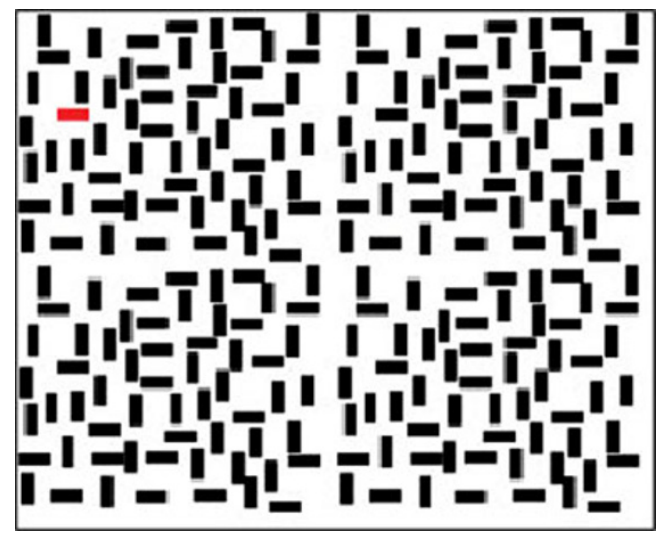

Fig. 4 The target is the one red horizontal line in the top left quadrant. The distracters are black horizontal and vertical lines. We can easily pick out the target based by its different color
On the other hand, in the second stage, selective attention and visual strategies based on top-down knowledge of the scene, such as searching for traffic signs along road sides and not in tree tops, make automatic predictions of search patterns difficult.

Based on the feature integration theory we can understand why we so easily detect the red line against all the black distracters in Fig. 4, but not among the red and black distracters in Fig. 5. In the first case, we can conduct a disjunctive parallel search based on one feature (color); in the second case we need to conduct a slow serial conjunctive search on both color (red) and shape (horizontal). The established way to measure this difference in saliency is using search time. The method is cumbersome. The subjects cannot know in advance where the target is

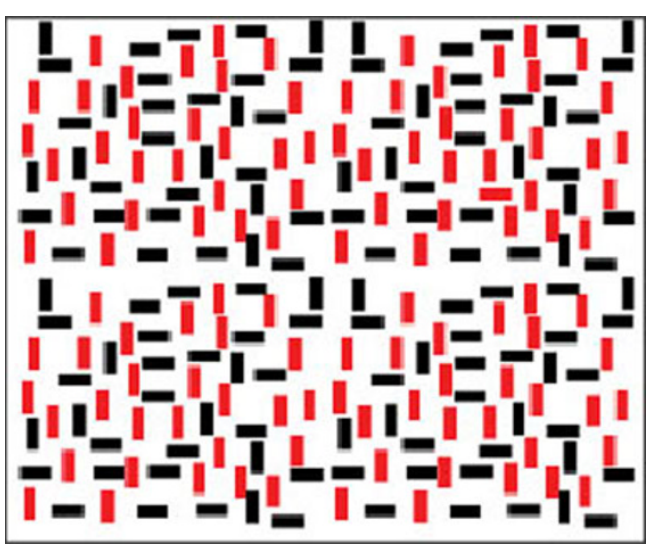

Fig. 5 In this panel about half of the lines are black and the other half are red. The black lines are both horizontal and vertical, the red lines are vertical. Only one of the red lines is horizontal - this is the target (top right quadrant). To spot the target both color and orientation needs to be processed 


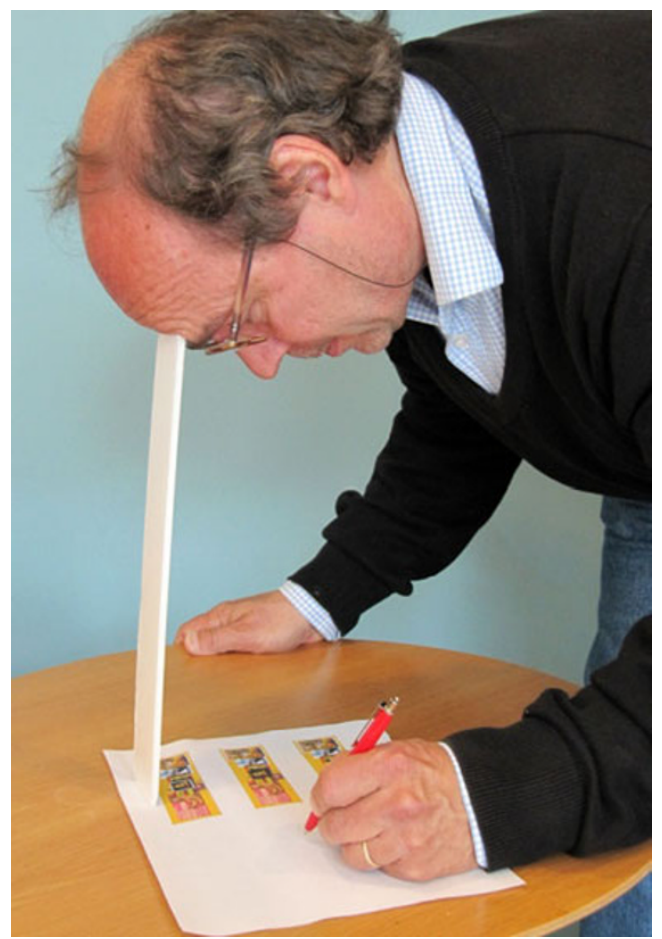

Fig. 6 One of the authors demonstrates how the measurements were conducted (here experiment 2): A $32 \mathrm{~cm}$ high support made sure the distance to the stimuli was the same for all participants. The subject fixated the tip of a ballpoint pen starting at the target sign and then moved the pen left away from the target until the target could no longer be seen in the corner of the eye. That point was then marked on the paper and the distance entered as the conspicuity index

but have to know what to look for, and each target can then only be used once. Furthermore, chance plays a role (subjects might happen to look right at the target at once), so a large number of subjects need to be used to reach significance. This is the background to Wertheim's suggested conspicuity index method.

\section{Conspicuity}

The method can be used both in the physical setting and on photographs of the scene. The subject fixates a point well to the side (or in any radial direction) of the target and then successively moves the gaze towards the target until it can be detected (detection conspicuity) or identified (identification conspicuity). The process is repeated three times and can also be done the other way, from the target out in any radial direction until detection (or identification) is no longer possible. The mean value of the angle can then be used as an index of conspicuity.

It is not self-evident that a measure of how well an object can be seen at different positions in the periphery of our vision can be applied to its visibility in the scene, but the correlation between conspicuity angle and traditional search times in natural scenes has been shown to be high [7, 8]. The logic is that during a search process, the larger the conspicuity index, the greater the chance that a target is spotted when a fixation is within the radius of the conspicuity angle. A study by Cook, Webber, Gillham, Le Scouiller, and Moseley [9] also showed good correlation with subjective ratings of visibility.

Theoretically, conspicuity is explained as based on a sensory phenomenon in the retina called lateral masking. This theory is in opposition to the feature integration theory and suggests that the slow search in conjunctive condition is due to lateral masking and not to focal attention. For a deeper theoretical explanation and experimental evidence, see Wertheim, Hooge, Krikke and Johnsson, [10].

\section{Method}

In this method section three experiments will be described. In the first experiment we wanted to find out if the difference in conspicuity, which intuitively was obvious in the two prepared stimulus in Fig. 3, with the sign against a contrasting green forest and a masking wall with posters and another sign, was measurable with the conspicuity index method suggested by Wertheim. And could it be done in a simple enough way so that the method could be used by information designers as a practical tool?

In second experiment we wanted to find out if the conspicuity index would react to small differences like

\section{Conspicuity index*}

(degrees)

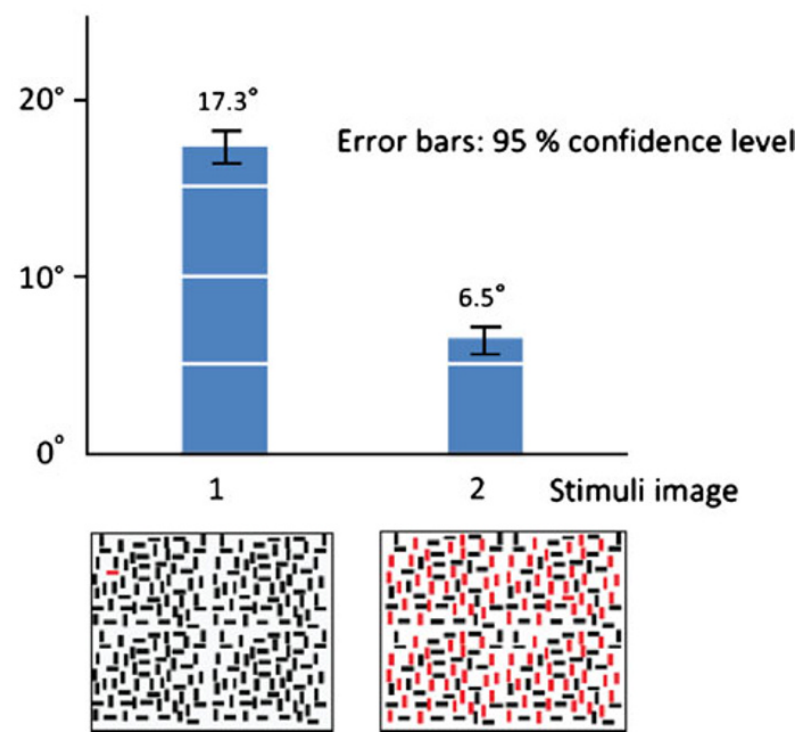

Fig. 7 The conspicuity index $\left(17.3^{\circ}\right)$ was much higher (signifying higher conspicuity) for the left panel with just black distracters, compared to the index $\left(6.5^{\circ}\right)$ of the right panel where the distracters were both red and black 
Table 1 Results from the conspicuity measurements of the panels in Fig. 3, 4 and 5

\begin{tabular}{lllll}
\hline & $\begin{array}{l}\text { Panel in } \\
\text { Fig. 4 }\end{array}$ & $\begin{array}{l}\text { Panel in } \\
\text { Fig. 5 }\end{array}$ & $\begin{array}{l}\text { Forest background } \\
\text { in Fig. 3 }\end{array}$ & $\begin{array}{l}\text { Wall background } \\
\text { in Fig. 3 }\end{array}$ \\
\hline $\mathrm{N}$ & 57 & 59 & 59 & 54 \\
Mean (mm) & 100 & 37 & $>135$ & 77 \\
SD (mm) & 20 & 17 & - & 25 \\
$95 \%$ Conf. level (mm) & 5 & 4 & - & 7 \\
Conspicuity angle (deg.) & 17.3 & 6.5 & $>42$ & 13.6 \\
95\% Conf. Level (deg.) & 0.9 & 0.8 & - & 1.2 \\
\hline
\end{tabular}

adding a mask behind the traffic sign in the previous experiment. A technique traditionally used on traffic and railway signals to enhance conspicuity.

In both of the experiments above the answer was intuitively obvious and we wanted to validate the conspicuity index method. In the third experiment we wanted to apply the method on a real problem on which we had no previous assumption.

\section{Experiment 1}

The two images in Fig. 3 and the two panels in Figs. 4 and 5 were used as stimuli. The images were printed on A4 paper where the image of each of the traffic sign were 100 by $28 \mathrm{~mm}$ (a boundary box precisely containing the drawing) and the two panels with red and black line segments were 58 by $70 \mathrm{~mm}$. They were all printed in color.

The paper with the stimuli was placed horizontally on a desk in a classroom environment with normal lighting conditions. A $32 \mathrm{~cm}$ tall support was placed on the paper slightly above the image area and was used as a headrest to ensure the same viewing distance for all subjects. The subjects were asked to rest their forehead on the support and view the drawing with both eyes as demonstrated in Fig. 6. They were to fixate the tip of a pen, which at the outset of the test, was pointed on the middle of the target sign. Then the subjects were asked to slowly move the pen horizontally to the left (for the panel in Fig. 4 to the right), away from the target, while continuing to fixate the tip of the pen. They were asked to continue moving the pen to the left until they could no longer detect the stimulus sign in the corner of their eye (while still fixating the tip of the pen). When they found a point where they could no longer detect the target they were asked to move the pen back and forward to further try to specify the location of this spot and then make a mark on the paper. This distance was later recalculated to an angle and used as the conspicuity index.

The panels were measured by 59 students, 35 male, 24 female, ages 23-30, from Austria, Holland, Sweden and Turkey. All had normal vision or vision corrected to normal. (In seven cases some panel was overlooked by participants, this is why the number of participants for these panels are lower than 59.)

\subsection{Results of experiment 1}

For the panel in Fig. 4, the subjects were asked to move their gaze to the right and mark the point where they could no longer detect the red horizontal line (among the black horizontal and vertical distracters). The distance between

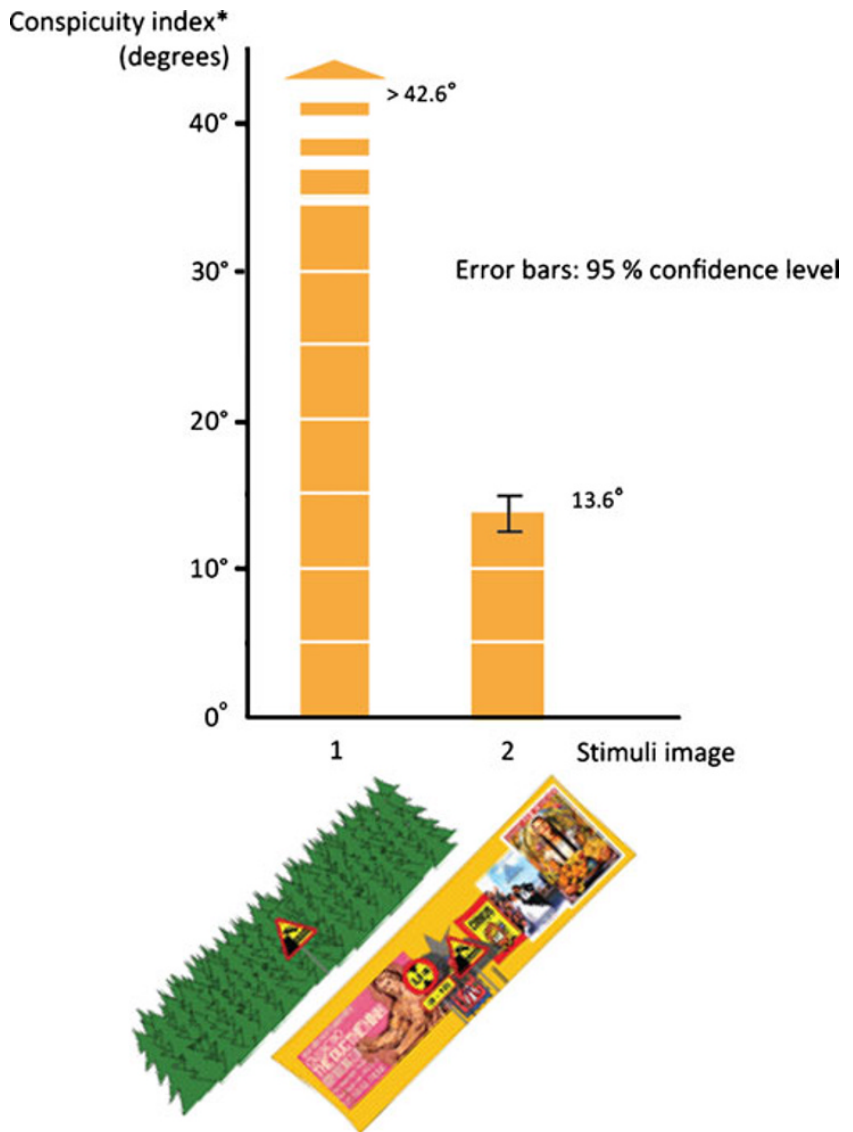

Fig. 8 The results of the conspicuity index method applied to the two drawings with the same sign against a green forest and against a similar colored and cluttered wall showed much higher index angle $\left(>42^{\circ}\right)$ compared to the other stimulus $\left(13.6^{\circ}\right)$ 
Fig. 9 A street sign in Prague, Czech Republic. A brightly colored mask has been added to the sign to enhance its visibility. The question is if enhancements by masks are measurable using the conspicuity index method?

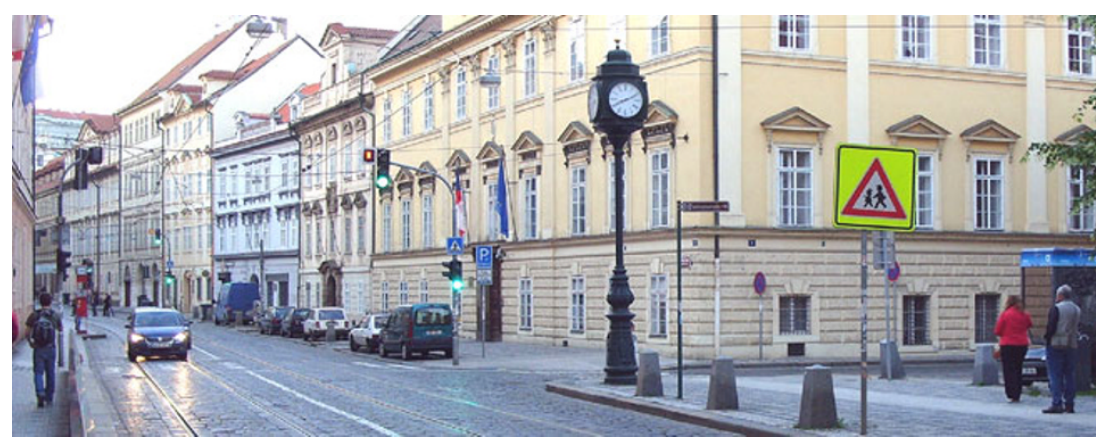

the left and mark the spot where they could no longer detect the presence of the traffic sign in question.

The results where clear and in accordance with the intuitive feeling that the target in the panel with red vertical and black horizontal and vertical distracters were more difficult to spot-lower conspicuity index - than the target in the panel with just black vertical and horizontal distracters. The distance between the target and the point
Fig. 10 The stimuli picture from experiment 1 (a) and with masks added (b-d)

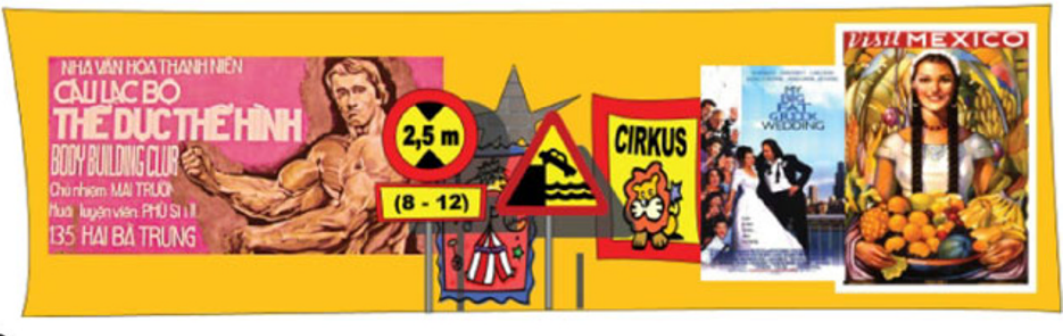

b

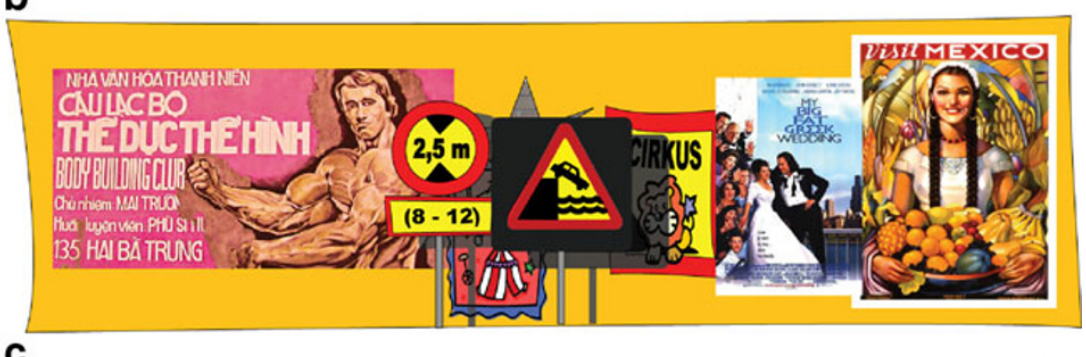

C

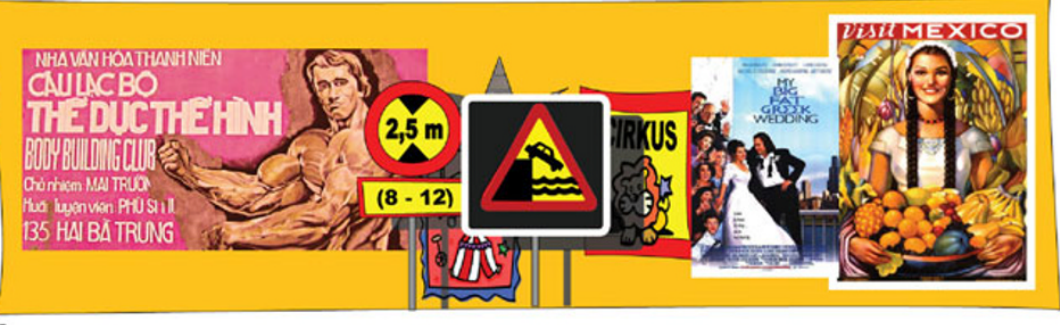

d

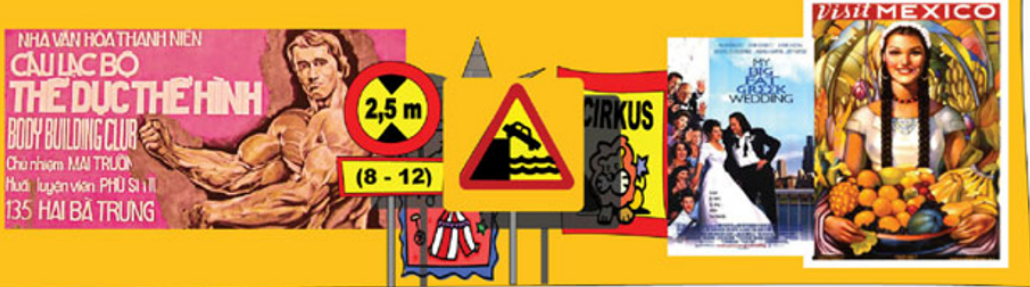


Fig. 11 The results of experiment 2 . There is a significant difference in the conspicuity index for stimuli $\mathrm{A}$, the sign against the cluttered wall, compared to stimuli $\mathrm{B}$, where the sign is enhanced by a black mask. There are differences between the three types of masks but they are not significant

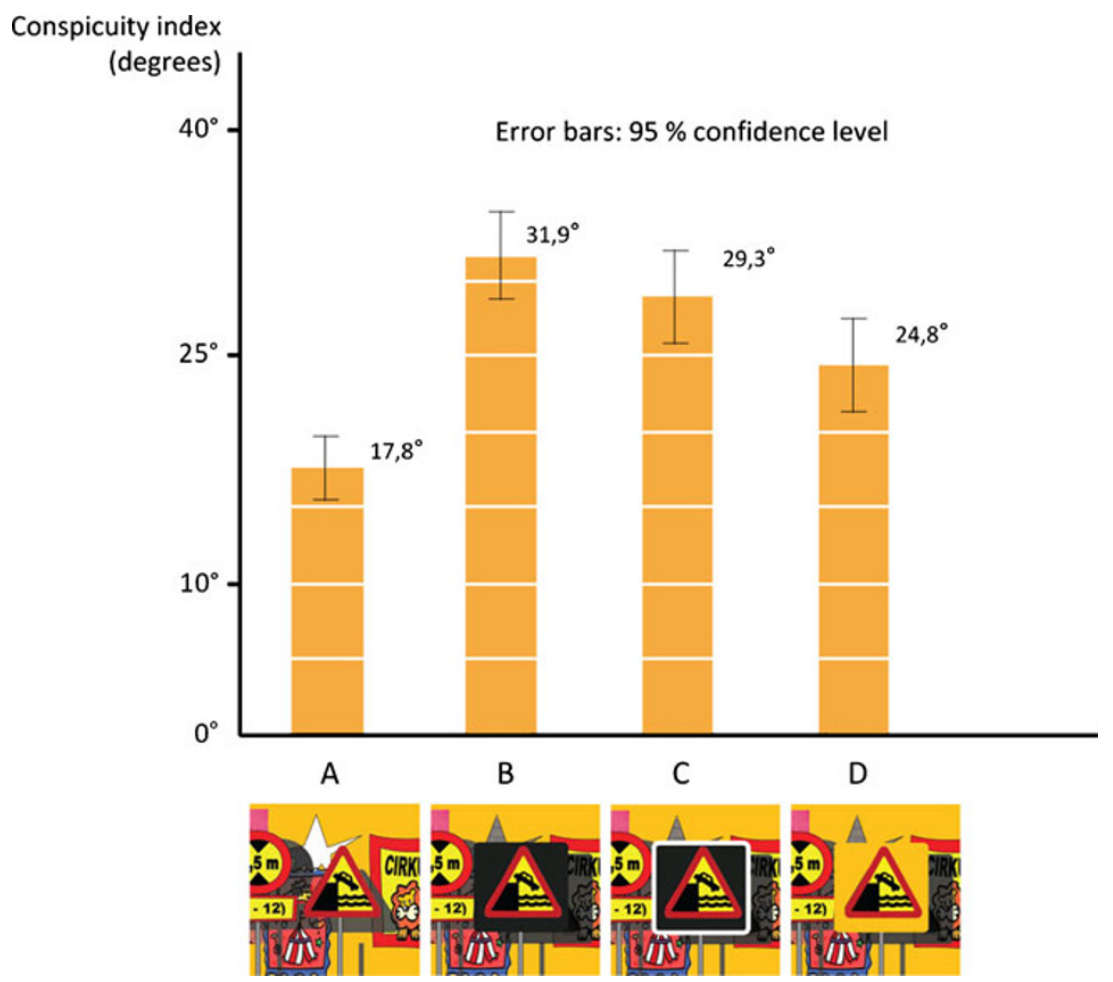

where the target could no longer be detected in the peripheral vision was much larger for the disjunctive panel. This distance was then recalculated to an angle measurement for the viewing distance of $32 \mathrm{~cm}$. See Fig. 7 and Table 1 for the results.

The results for the prepared stimulus with the traffic sign against a green forest and a cluttered yellow wall showed the same clear results when measured with the conspicuity index method: the picture with the sign against the green forest had such a large index that the point where the sign could no longer be detected was way outside of the A4 paper used to mark the index point. Thus we know that the index point was more than $135 \mathrm{~mm}$ (more than $42^{\circ}$ ), but were not able to record it with the simplified technique used in this experiment. The stimuli with the sign against the cluttered wall had a conspicuity index of $13.6^{\circ}$. See Fig. 8 and Table 1.

The results were very clear and consistent and the variation surprisingly small. We think the results clearly show that the intuitively obvious difference in conspicuity also could be measured using the method.

The interesting question was now whether small differences were also possible to measure using this method (Fig. 9).

\section{Experiment 2}

In the second experiment we wanted to see if the traditional method of increasing the conspicuity of traffic signals by adding a black mask behind the sign was measurable using the conspicuity indexing method.

We used one of the picture from experiment 1, with the traffic sign against the cluttered wall (Fig. 10a), and added a black mask (Fig. 10b). We also used two other variations of the mask (black with a white border and plain yellow, like the background wall- - see Fig. 10c and d).

This time the stimuli pictures where slightly larger, 115 by $34 \mathrm{~mm}$, and placed to the right on an A4 paper in landscape orientation (see Fig. 6). The participants used a carton support to make sure the distance between the eye and the stimuli was approximately $32 \mathrm{~cm}$. In this study the 13 Swedish students participated (age 25-30, gender was not recorded). All subjects had normal eyesight, or eyesight

The expectation was that this mask would increase the conspicuity angle by making the sign more conspicuous.

Table 2 Conspicuity indices for experiment 2

\begin{tabular}{lllll}
\hline & A & B & C & D \\
\hline N & 13 & 13 & 13 & 13 \\
Mean (mm) & 97,8 & 169,4 & 156,8 & 134,2 \\
SD (mm) & 23,6 & 29,9 & 31,7 & 31,4 \\
95\% conf. level (mm) & 13,3 & 16,3 & 17,2 & 17,1 \\
Conspicuity angle (deg.) & 17,8 & 31,9 & 29,3 & 24,8 \\
95\% conf. level (deg.) & 2,4 & 2,9 & 3,1 & 3,1 \\
\hline
\end{tabular}
corrected to normal. 
Fig. 12 A school bus in Eskilstuna, Sweden. The visibility of the bus is high in contrast to the winter snow but the school bus sign is partly camouflaged through the color of the bus, which happens to be of the same color as the sign

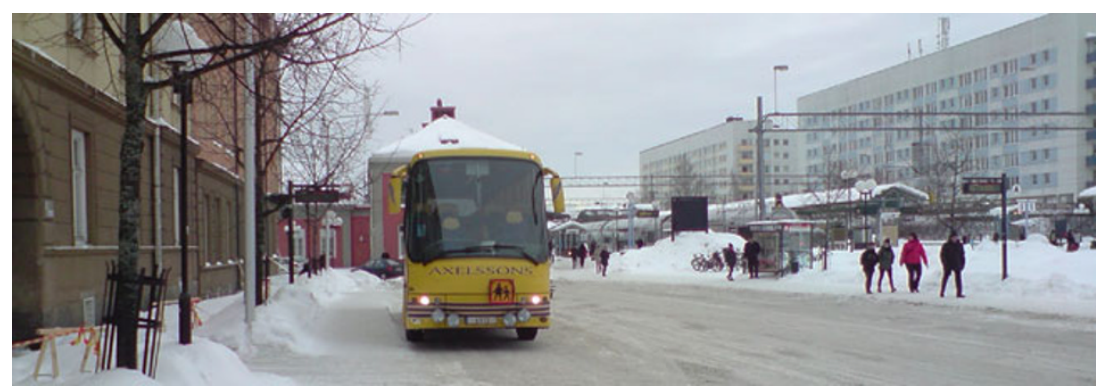

\subsection{Results of experiment 2}

The results showed a significant difference in conspicuity index between stimuli $\mathrm{A}$, (the sign without any mask), and stimuli $\mathrm{B}, \mathrm{C}$, and $\mathrm{D}$, with masks. The conspicuity index angle for stimuli A was $17.8^{\circ}$ and for stimuli B, with the plain black mask, $31.9^{\circ}$. The yellow mask with the same color as the background had the lowest index angle. The differences between the three different masks were not significant. The variance between subjects was still very low with the $95 \%$ confidence levels at around $3^{\circ}$. The results are presented in Fig. 11 and in Table 2.

The results of experiment 1 and 2 were reassuring. It looked as if the conspicuity index could not only be measured with the simple method used, but also that it was sensitive enough to measure relatively small differences.

In the final experiment we wanted to use the method on a real problem in an ongoing project: to test differences in visibility of different design for school bus signs (Fig. 12).

\section{Experiment 3}

Every year a large number of school children are killed in accidents relating to school bus transportation (Fig. 12). This problem is addressed in the EU project Safeway two School involving a number of European nations. One central question in this project is: How do we make school bus signs visible? Our task in this project was to help determine which of several tested school bus signs were most visible, that is had the highest conspicuity index.

This time a more elaborated system was used which allowed for a higher degree of control. The stimulus was presented on a monitor. The screen's physical dimensions were 523 by $327 \mathrm{~mm}$ and the resolution 1920 by 1,200 pixels. The subject was seated in front of the monitor using a chin rest fixating the viewing distance to $660 \pm 10 \mathrm{~mm}$.

As a background to the different bus signs tested the manipulated traffic environment in Fig. 13 was used. Parts of the picture were copied twice to make it wide enough. The background was a semi realistic view that would act as a mask for all the tested signs, allowing us to make some kind of comparison between their conspicuity. To the far right of the background picture the target stimulus was shown added to the back of a gray bus. The size of the sign corresponded to a real world size of 500 by $500 \mathrm{~mm}$.

On the monitor screen the size of the sign was 48 by 48 pixels or 13 by $13 \mathrm{~mm}$ constituting a viewing angle of 1.13 by $1.13^{\circ}$. In real life this corresponded to looking at the bus sign from a distance of about $25 \mathrm{~m}$.

The measurements were this time done using a small yellow dot as a fixation mark for the participant (barely seen in the back windscreen of the rightmost gold colored
Fig. 13 The stimuli picture with the target (the bus sign) to the far right, at the lower right corner of the bus. During the test the participants were to fixate on a "gaze point" in the form of a yellow dot that could be moved left and right by the participant. In this screen dump it can barely be seen in the middle of the back windscreen of the rightmost gold colored car

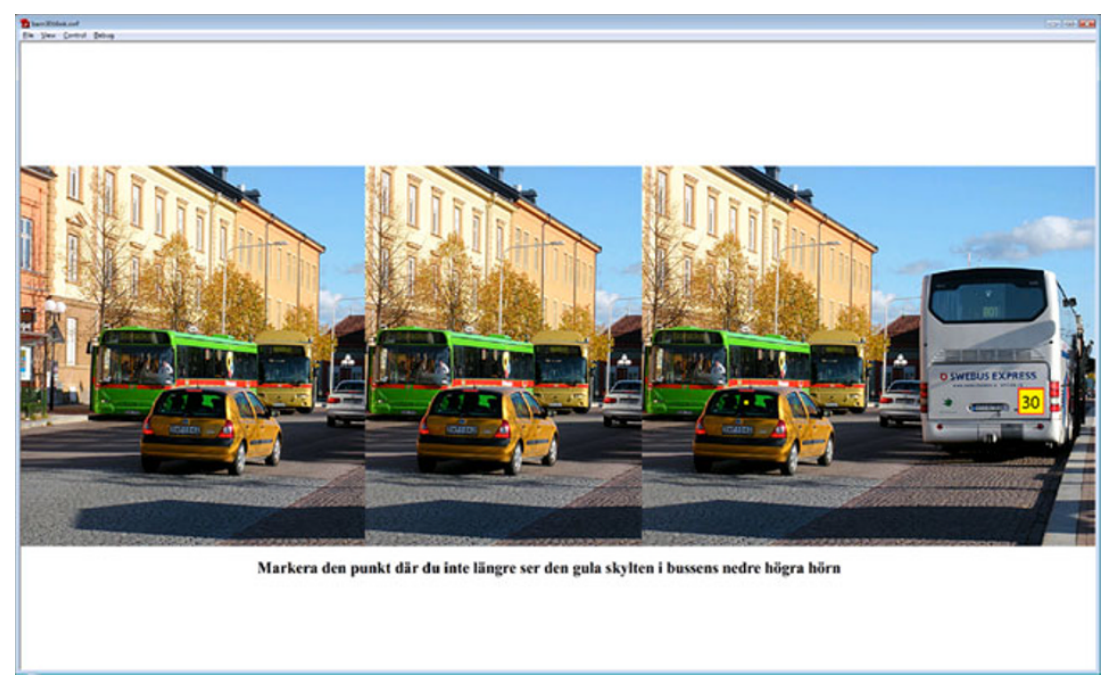


Table 3 The stimuli signs used in experiment 3

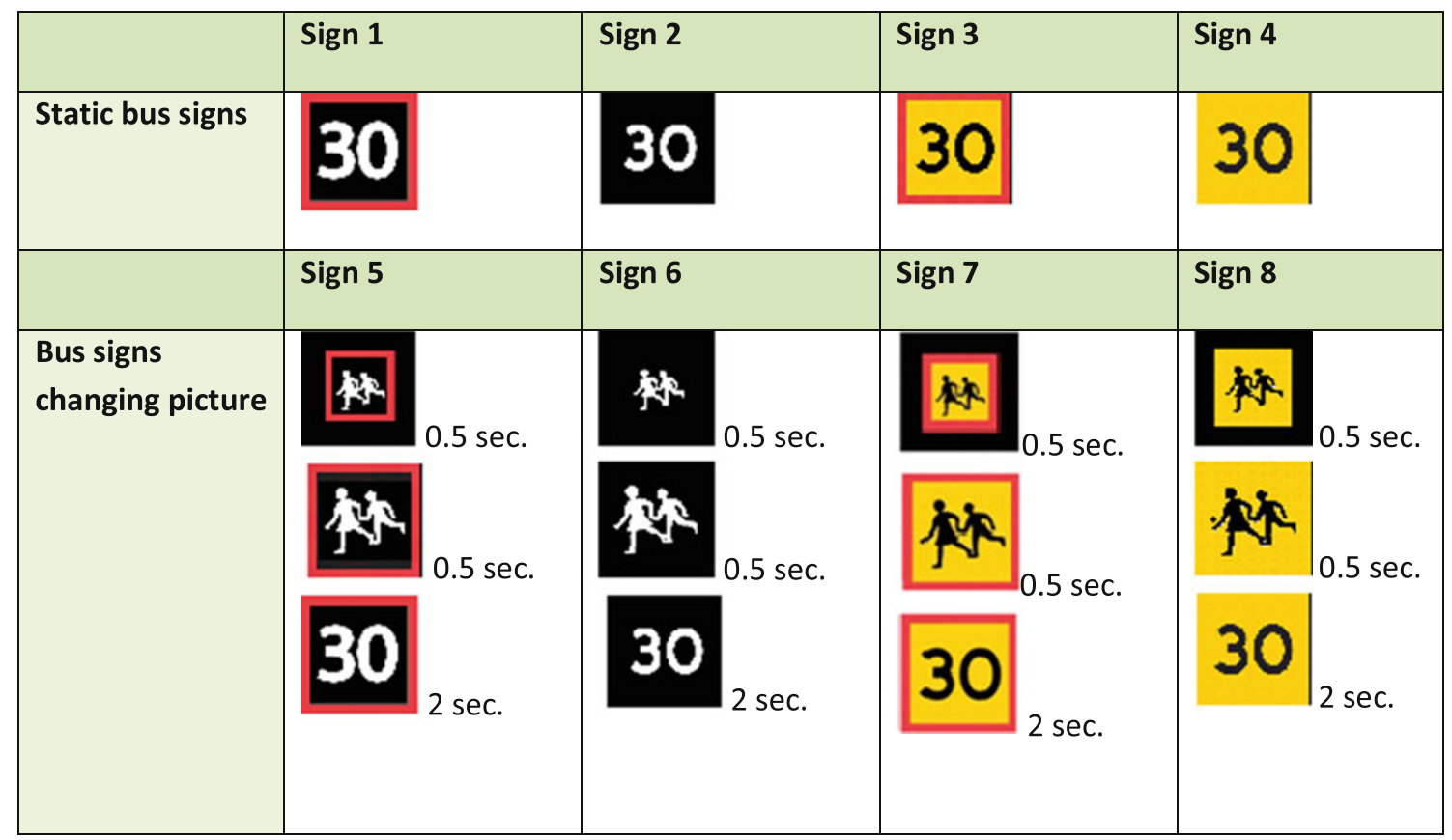

car in Fig. 13). The dot could be moved left or right in small 5 pixel increments by the participant using the left and right arrow keys on a keyboard. At the outset of the first trial the dot was on top of the bus sign. The participant would then move the dot gradually to the left. Fixating it with his or her gaze until the bus sign could no longer be seen in the corner of the eye. The participant would then mark the distance by pressing the space bar. The dot would then move to the far left in the picture and the procedure would restart using the same stimulus but this time moving right until the participant was able to distinguish the bus sign in the corner of the eye. This went on three times starting from the sign and three times staring from the far left, for each stimulus. The mean value of the six trials was then used as measurement.

Eight different bus signs were used as stimuli. Four static and four others where the picture changed during a time interval of $3 \mathrm{~s}$ (see Table 3).

Based on the low variance in the previous experiments only five subjects were used for this test (males 27 to
56 years of age). All subjects had normal eyesight, or eyesight corrected to normal.

\subsection{Results for experiment 3}

The results showed a significant difference between the conspicuity index of the two groups of static bus signs (about $20-25^{\circ}$ ) and picture changing signs ( 35 to $36^{\circ}$ ). The picture changing signs (sign 5-8) showed higher conspicuity index, they were thus more visible (see Fig. 14 and Table 4). However the differences within the four picture changing signs were small and not significant. The differences within the group of static signs were also small and not significant.

There was a larger variance between participants in this experiment. The point of detection seemed to be more difficult to establish when asking the participants. If this has something to do with the new method displaying the stimuli on a monitor as opposed to printed on a paper is something that we will need to investigate further in future tests. We
Table 4 Results from experiment 3

\begin{tabular}{lllllllll}
\hline & Sign 1 & Sign 5 & Sign 2 & Sign 6 & Sign 3 & Sign 7 & Sign 4 & Sign 8 \\
\hline $\mathrm{N}$ & 5 & 5 & 5 & 5 & 5 & 5 & 5 & 5 \\
Mean (px) & 934 & 1730 & 898 & 1721 & 1039 & 1771 & 1115 & 1753 \\
SD (px) & 426 & 236 & 333 & 266 & 369 & 218 & 517 & 213 \\
95\% conf. Level (px) & 373 & 207 & 292 & 233 & 323 & 191 & 453 & 187 \\
Index (degrees) & 20,9 & 35,3 & 20,2 & 35,1 & 23,0 & 35,9 & 24,5 & 35,6 \\
SD (degrees) & 9,9 & 5,5 & 7,8 & 6,2 & 8,6 & 5,1 & 11,9 & 5,0 \\
95\% conf. level & 8,7 & 4,8 & 6,8 & 5,4 & 7,5 & 4,5 & 10,4 & 4,4 \\
\hline
\end{tabular}


Fig. 14 The results of experiment 3 show that the picture changing signs were more conspicuous than the static sign. The differences within the two groups of dynamic and static signs were small and not significant

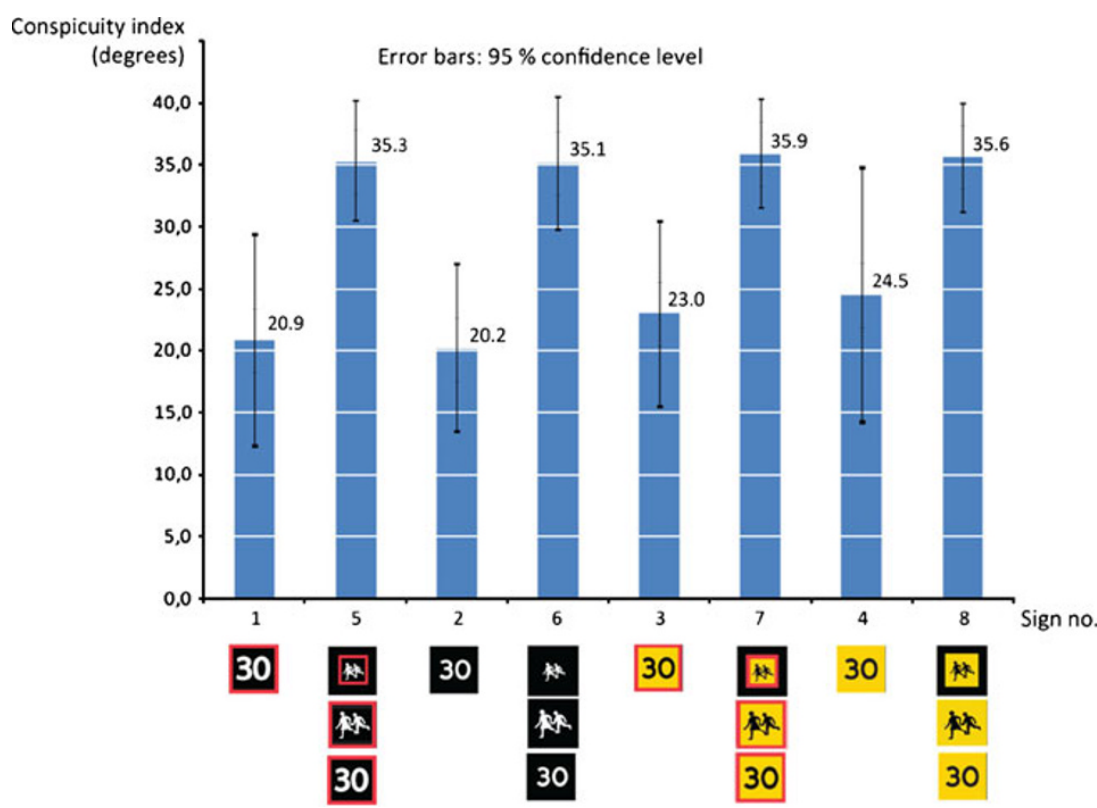

also noticed a small difference between the index when the participants started with the gaze on the target and went outward, than when the procedure was the reversed. However, the differences between signs were not affected.

Considering the higher variance we can conclude that the number of participants (5) was too low in experiment 3. We also need to work on optimizing the method with the monitor interface.

\section{Discussion}

The simple experiment method used in experiment 1 and 2 showed robust results suggesting that the conspicuity index method can be used to measure visibility of signs in their context. The results showed low variations across subjects, suggesting that fewer subjects could be used than in the first experiment. The results also showed good correlation with intuitive expectations. Experiment 2 also suggested that small differences are detectable using this method. The results of experiment 3 were less robust, showing a higher variance between participants for the same sign. If this has something to do with the new method introduced here displaying the stimuli on a monitor screen or with the relatively few participants used will be the subject of future studies.

One has to be aware of the fact that the visibility of the sign now is measured in context and that the same sign against another background will show different results. Thus it is the relative values that are of interest not the absolute ones.

However, the ultimate goal for the development of this method would be to be able to define a method where the conspicuity index of different signs could be tested against one, or several standard backgrounds and an index threshold decided below which no traffic sign would be considered visible enough. If lower, something would have to be done about the context, using masks, separating large clusters of signs, etc. This will also be the subject of future studies.

\section{Conclusions}

The results from this study show that Wertheims conspicuity index method is useful in determining the visibility of signs in context. The simple method with the stimuli printed on paper used in experiment 1 and 2 should be valuable for practical use by designers in an everyday setting. The robustness of the results found suggests that a relatively low number of participants still could give useful results. The results from the last experiment using even fewer participants and a new computer based method were less robust and needs to be investigated further.

As a guideline to sign design the quantitative results from this study show that uncluttering of the immediate background of signs by using a mask clearly increases the conspicuity of the sign.

Acknowledgement Parts of his study has been conducted within the $\mathrm{EU} 7^{\text {th }}$ Framework program SAFEWAY 2 SCHOOL, which is gratefully acknowledged.

Open Access This article is distributed under the terms of the Creative Commons Attribution License which permits any use, distribution and reproduction in any medium, provided the original author(s) and source are credited. 


\section{References}

1. Wertheim AH (1989) A quantitative conspicuity index: theoretical foundation and experimental validation of a measurement procedure. Report C-20 (in Dutch). TNO Human Factors Research Institute, Soesterberg, The Netherlands

2. Purves D, Augustine GJ, Fitzpatrick D, Katz LC, LaManta A-S, Williams SM (2001) Neuroscience. Sinauer, Sunderland

3. Itti L, Koch C (2000) A saliency-based search mechanism for overt and covert shifts of visual attention. Vis Res 40:1489-1506

4. Simons DJ, Levin DT (1997) Failure to detect changes to attended objects. Investig Ophthalmol Vis Sci 38:3273

5. Treisman A, Gelade G (1980) A feature integration theory of attention. Cognit Psychol 12:97-136
6. Koch C, Ullman S (1985) Shifts in selective visual attention: towards the underlying neural circuitry. Hum Neurobiol 4:219-227

7. Kooi FL, Toet A (1999) Conspicuity: an efficient alternative for search time. In: Gale AG, Brown ID, Hasslegrave CM, Taylor SP (eds) Vision in vehicles VII. Elsevier, Amsterdam, pp 451-462

8. Toet A, Kooi FL, Bijl P, Valeton JM (1998) Visual conspicuity determines human target acquisition performance. Opt Eng 37 (7):1969-1975

9. Cook G, Webber G, Gillham SA, Le Scouiller SA, Moseley E (2005) The legibility and conspicuity of emergency escape route signage for people with visual impairments. Int Congr Series 1282:1016-1020

10. Wertheim AH, Hooge LTC, Krikke K, Johnsson A (2006) How important is lateral masking in visual search? Exp Brain Res $170: 387-402$ 